\title{
Serum bilirubin concentration is modified by UGT1A1 Haplotypes and influences risk of Type-2 diabetes in the Norfolk Island genetic isolate
}

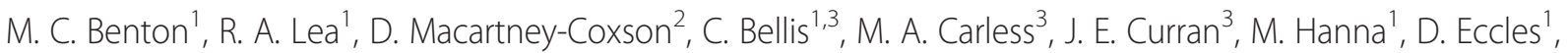 \\ G. K. Chambers ${ }^{4}$, J. Blangero ${ }^{5}$ and L. R. Griffiths ${ }^{1 *}$
}

\begin{abstract}
Background: Located in the Pacific Ocean between Australia and New Zealand, the unique population isolate of Norfolk Island has been shown to exhibit increased prevalence of metabolic disorders (type-2 diabetes, cardiovascular disease) compared to mainland Australia. We investigated this well-established genetic isolate, utilising its unique genomic structure to increase the ability to detect related genetic markers. A pedigree-based genome-wide association study of 16 routinely collected blood-based clinical traits in 382 Norfolk Island individuals was performed.

Results: A striking association peak was located at chromosome 2 q37.1 for both total bilirubin and direct bilirubin, with 29 SNPs reaching statistical significance $\left(P<1.84 \times 10^{-7}\right)$. Strong linkage disequilibrium was observed across a $200 \mathrm{~kb}$ region spanning the UDP-glucuronosyltransferase family, including UGTIA1, an enzyme known to metabolise bilirubin. Given the epidemiological literature suggesting negative association between CVD-risk and serum bilirubin we further explored potential associations using stepwise multivariate regression, revealing significant association between direct bilirubin concentration and type-2 diabetes risk. In the Norfolk Island cohort increased direct bilirubin was associated with a $28 \%$ reduction in type-2 diabetes risk (OR: $0.72,95 \% \mathrm{Cl}: 0.57-0.91, P=0.005)$. When adjusted for genotypic effects the overall model was validated, with the adjusted model predicting a $30 \%$ reduction in type- 2 diabetes risk with increasing direct bilirubin concentrations (OR: 0.70, $95 \%$ Cl: 0.53-0.89, $P=0.0001$ ).

Conclusions: In summary, a pedigree-based GWAS of blood-based clinical traits in the Norfolk Island population has identified variants within the UDPGT family directly associated with serum bilirubin levels, which is in turn implicated with reduced risk of developing type-2 diabetes within this population.
\end{abstract}

Keywords: Norfolk Island, GWAS, Bilirubin, type-2 diabetes, UGT1A1

\section{Background}

This study examined a large multi-generational pedigree from the isolated population of Norfolk Island to identify genomic variants (SNPs - single nucleotide polymorphisms) associated with routinely collected blood-based clinical traits. The Norfolk Island population is a genetic isolate with strong family groups and a well-documented

\footnotetext{
*Correspondence: lyn.griffiths@qut.edu.au

${ }^{1}$ Genomics Research Centre, Institute of Health and Biomedical Innovation, Queensland University of Technology, Kelvin Grove, QLD 4059, Australia Full list of author information is available at the end of the article
}

family genealogy [1]. Norfolk Island is a small volcanic island located in the Pacific Ocean between Australia (about $1600 \mathrm{~km}$ north-east of Sydney) and New Zealand (1077 km north-west of Auckland). Alongside geographic isolation, a unique history has shaped the genomic architecture of the current pedigree members resulting in an admixed population with both European and Polynesian ancestry [2]. Recent estimation of the admixture in the Norfolk Island cohort reported 88 \% European ancestry and $12 \%$ Polynesian ancestry [2].

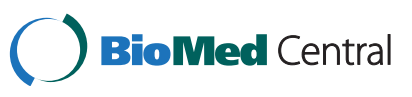

(c) 2015 Benton et al. Open Access This article is distributed under the terms of the Creative Commons Attribution 4.0 International License (http://creativecommons.org/licenses/by/4.0/), which permits unrestricted use, distribution, and reproduction in any medium, provided you give appropriate credit to the original author(s) and the source, provide a link to the Creative Commons license, and indicate if changes were made. The Creative Commons Public Domain Dedication waiver (http://creativecommons.org/publicdomain/zero/1.0/) applies to the data made available in this article, unless otherwise stated. 
To date the Norfolk Island Health Study (NIHS) has collected data and samples for 1199 Norfolk Islanders, $52 \%(N=624)$ of whom were found to have direct links to the original founders. Using this in-depth genealogical information a large multi-generational Norfolk pedigree was reconstructed [1]. Several studies have established admixture scores and presence of founder effects within the Norfolk Island pedigree [1-3] and the pedigree has been shown to have sufficient power to detect genetic loci influencing complex traits via linkage and association [4-7].

The Norfolk Island population has high rates of metabolic syndrome [7] and cardiovascular related risk factor traits, especially obesity, compared to mainland Australia. Research on the Norfolk pedigree has shown that traits for obesity, dyslipidaemia, blood glucose and hypertension exhibit a substantial genetic component, with heritability estimates ranging from $30 \%$ for systolic blood pressure (SBP) to $63 \%$ for low density lipoproteins (LDL) cholesterol $[1,4,5]$. In addition, factor analysis identified "composite" phenotypes with high heritability [5], suggesting that common gene(s) underlie cardiovascular diseaserelated phenotypes. Furthermore, genetic linkage analysis in the Norfolk Island pedigree has successfully identified previously documented regions associated with cardiovascular disease risk traits, the most significant being for SBP on chromosome 1 (1p36) [4].

Reported rates of type- 2 diabetes within the Norfolk Island population are similar to mainland Australia (4$8 \%$ ). However, a significantly higher proportion of individuals had fasting blood glucose in excess of normal ranges $(>5 \mathrm{mmol} / \mathrm{L}$ ), suggesting a high prevalence of pre-diabetes and possible under-diagnosis of type- 2 diabetes [4, 8]. Additionally, clinical diagnosis of type- 2 diabetes using AUSDRISK [9] identified that $42 \%$ of the Norfolk Island population were at high-risk of developing the disease [7].

Bilirubin is a component of haemoglobin, formed during metabolic breakdown in the liver. Total serum bilirubin measures both water-soluble (direct-) and fat-soluble (indirect-) bilirubin. Bilirubin is also a potent antioxidant and as such has a vital role in the protection of the body against reactive oxygen species [10-12]. Numerous epidemiological analyses have reported strong negative associations between CVD-risk and serum bilirubin levels. Very few studies investigating the link between type-2 diabetes and serum bilirubin concentration have been conducted [13], although recently an association with mortality in a type-2 diabetic cohort was observed [14]. Serum bilirubin concentration has been shown to be tightly regulated by the UDP-glucuronosyltransferase (UDPGT) enzyme family, with several large GWAS and linkage studies identifying variants within UGT1A in particular [15-18]. This is suggestive of a potentially heritable metabolic disease factor, for which a recent study provides further supportive evidence; a Mendelian randomization study exploring total bilirubin levels in a prospective study found further evidence for a protective role in type- 2 diabetes [19].

The aim of this study was to update the previously calculated heritabilities for a range of blood-based traits relating to CVD risk in the Norfolk Island cohort and to perform genome-wide association studies (GWASs) of the heritable traits using a pedigree-based approach.

\section{Results}

\section{Heritability of individual metabolic traits}

A description of the blood-based clinical traits investigated in this study, including summary statistics, is shown in Additional file 1. The latest pedigree relationship information and GenABEL were used to calculate heritability $\left(\mathrm{h}^{2}\right)$ statistics for all traits profiled in the Norfolk Island cohort. In total, 16 traits (out of 19) yielded statistically significant $\mathrm{h}^{2}$ values ranging from $0.225-0.563$ (nominal $P<0.05)$. The average heritability was 0.39 and 8 traits exhibited a higher than average heritability (total protein, globin, total bilirubin, LDL-C, cholesterol, alkaline phosphatase, and urea) the most heritable trait being total protein $\left(\mathrm{h}^{2}=0.563, P=2.26 \times 10^{-4}\right)$. A summary of all significantly heritable major blood-based clinical traits is shown in Table 1.

\section{GWAS of metabolic traits}

All 16 heritable blood-based clinical traits were screened for association separately; individual trait GWAS Manhattan plots can be viewed in Additional file 2. There were 2 traits with robustly associated clusters (i.e. SNPs in close

Table 1 Significantly heritable metabolic traits in the Norfolk Island population

\begin{tabular}{lll}
\hline Trait & $h^{2}$ & $P$ value \\
\hline Total protein & 0.563 & $2.26 \mathrm{E}-04$ \\
Globin & 0.525 & $3.36 \mathrm{E}-04$ \\
Total bilirubin & 0.502 & $4.45 \mathrm{E}-05$ \\
LDL-C & 0.454 & $7.51 \mathrm{E}-05$ \\
Cholesterol & 0.426 & $8.41 \mathrm{E}-05$ \\
Chlorine & 0.426 & $8.41 \mathrm{E}-05$ \\
Alkaline phosphatase & 0.425 & $6.10 \mathrm{E}-04$ \\
Urea & 0.424 & $7.87 \mathrm{E}-04$ \\
GGT & 0.369 & $3.02 \mathrm{E}-03$ \\
Albumin & 0.358 & $9.01 \mathrm{E}-03$ \\
Uric Acid & 0.350 & $2.17 \mathrm{E}-03$ \\
HDL-C & 0.348 & $1.58 \mathrm{E}-02$ \\
Direct Bilirubin & 0.327 & $3.30 \mathrm{E}-02$ \\
Creatinine & 0.257 & $2.43 \mathrm{E}-02$ \\
AST & 0.251 & $3.81 \mathrm{E}-02$ \\
Cholesterol/HDL-C ratio & 0.225 & $2.42 \mathrm{E}-02$ \\
\hline
\end{tabular}


proximity to each other); total bilirubin and direct bilirubin. It should be noted that a number of SNPs passed the adjusted significance threshold for liver function traits (i.e. GGT, AST, ADH). These traits exhibited numerous SNPs passing $M_{\text {eff }}$ adjustment, however robust 'peaks'/clusters of SNPs were not observed.

\section{Exploration of the bilirubin association on chromosome 2q37.1}

The strongest observed association was seen between a cluster of 29 SNPs on chromosome 2 q37.1 passing a $M_{\text {eff }}$ adjusted threshold and total serum bilirubin (Fig. 1a, Table 2). The most robustly associated SNP was rs6744284 $\left(\mathrm{P}=1.87 \times 10^{-16}\right)$. A weaker association was observed for the same cluster of SNPs on chromosome 2q37.1 with direct serum bilirubin levels (Fig. 1b). These 29 SNPs span a region of $189.8 \mathrm{~kb}$, and lie directly on top of a complex locus that codes numerous isoforms of the UDPglucuronosyltransferase (UGT) family (Fig. 2).

\section{LD block identification}

Evidence of strong linkage disequilibrium (LD) across the 29 SNPs was observed in the Norfolk Island population (Fig. 3); summarised LD statistics for the 29 SNPs: $\mathrm{r}^{2}(\min =0.026$, 1st Quartile $=0.33$, median $=$ 0.49 , mean $=0.51$, 3rd Quartile $=0.72, \max =1.00)$, D' $(\min =0.24,1$ st $Q u a r t i l e=0.82$, median $=0.90$, mean $=$ 0.89 , 3rd Quartile $=1.00, \max =1.00$ ). . Haploview analysis identified 2 LD blocks across the region; the first block contained 9 SNPs and spanned $88 \mathrm{~kb}$, the second block consisted of 19 SNPs and spanned a region of $74 \mathrm{~kb}$. Further analysis of LD across 3 separate HapMap populations was conducted to compare with that obtained in the Norfolk Island cohort; CEU (European), CHD (Chinese) and JPT (Japanese). Due to the use of different SNP arrays, 25 of the 29 SNPs were available across the 4 populations, thus the LD mapping was restricted to these 25 SNPs. The LD pattern for the Norfolk Island cohort was most similar to the CEU population, and extensively different from both of the Asian HapMap groups used (Additional file 3). LD

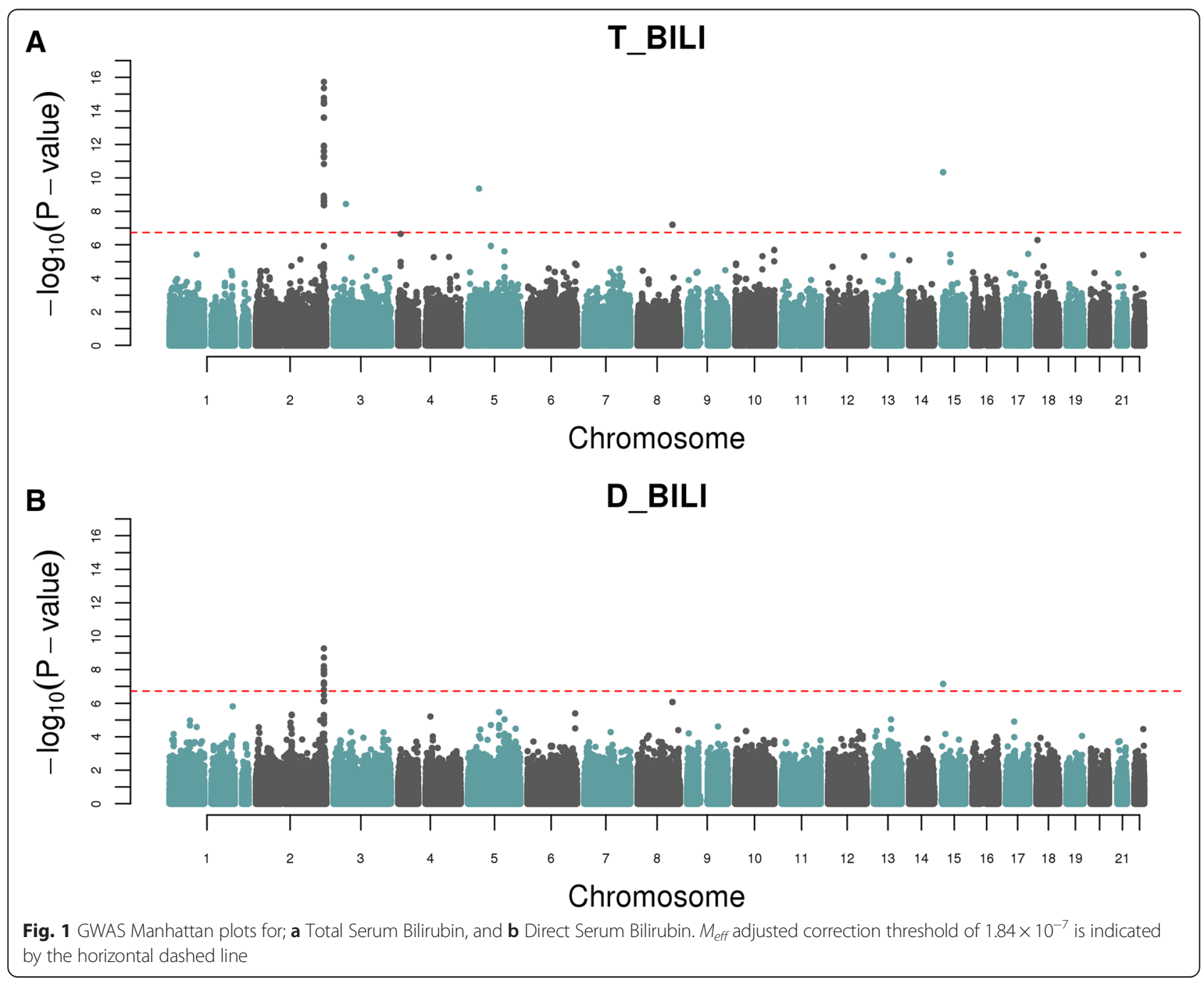


Table 2 Top $M_{\text {eff }}$ corrected SNPs associated with total serum bilirubin

\begin{tabular}{|c|c|c|c|c|c|c|c|c|}
\hline SNP & $\mathrm{CHR}$ & Position & $\mathrm{A} 1$ & A2 & effB & se_effB & chi2.1df & $P$ value \\
\hline rs6744284 & 2 & 234625297 & $C$ & $\mathrm{~T}$ & 3.278 & 0.395 & 68.90 & $1.87 \mathrm{E}-16$ \\
\hline rs3771341 & 2 & 234673239 & C & $\mathrm{T}$ & 3.235 & 0.395 & 67.17 & 4.43E-16 \\
\hline rs17863787 & 2 & 234611094 & $\mathrm{~T}$ & G & 3.044 & 0.379 & 64.48 & 1.70E-15 \\
\hline rs6742078 & 2 & 234672639 & G & $\mathrm{T}$ & 3.025 & 0.379 & 63.60 & 2.64E-15 \\
\hline rs887829 & 2 & 234668570 & G & A & 3.011 & 0.379 & 63.01 & 3.55E-15 \\
\hline rs4148324 & 2 & 234672722 & $\mathrm{~T}$ & G & 3.011 & 0.379 & 63.01 & $3.55 \mathrm{E}-15$ \\
\hline rs4148325 & 2 & 234673309 & C & $\mathrm{T}$ & 3.011 & 0.379 & 63.01 & 3.55E-15 \\
\hline rs1105880 & 2 & 234601965 & $\mathrm{~T}$ & C & 2.829 & 0.368 & 59.13 & $2.46 \mathrm{E}-14$ \\
\hline rs1105879 & 2 & 234602202 & $\mathrm{~T}$ & G & 2.829 & 0.368 & 59.13 & 2.46E-14 \\
\hline rs6725478 & 2 & 234615400 & C & $\mathrm{T}$ & 2.711 & 0.378 & 51.40 & $1.18 \mathrm{E}-12$ \\
\hline rs 2741045 & 2 & 234580140 & C & $\mathrm{T}$ & 2.907 & 0.406 & 51.17 & 1.32E-12 \\
\hline rs10168416 & 2 & 234597087 & C & G & 2.827 & 0.400 & 49.85 & $2.55 \mathrm{E}-12$ \\
\hline rs2070959 & 2 & 234602191 & A & G & 2.827 & 0.400 & 49.85 & $2.55 \mathrm{E}-12$ \\
\hline rs 2741012 & 2 & 234508963 & C & $\mathrm{T}$ & 2.887 & 0.415 & 48.43 & $5.20 \mathrm{E}-12$ \\
\hline rs 2741027 & 2 & 234518011 & G & A & 2.887 & 0.415 & 48.43 & $5.20 \mathrm{E}-12$ \\
\hline rs7608175 & 2 & 234599089 & C & G & 2.536 & 0.364 & 48.41 & $5.27 \mathrm{E}-12$ \\
\hline rs10168155 & 2 & 234596836 & C & $\mathrm{T}$ & 2.530 & 0.364 & 48.21 & $5.82 \mathrm{E}-12$ \\
\hline rs10171367 & 2 & 234597667 & C & G & 2.530 & 0.364 & 48.21 & $5.82 \mathrm{E}-12$ \\
\hline rs2361502 & 2 & 234698790 & $\mathrm{~T}$ & C & 2.590 & 0.380 & 46.37 & 1.46E-11 \\
\hline rs 2925260 & 15 & 25869972 & $\mathrm{~T}$ & C & 18.764 & 2.825 & 44.11 & 4.56E-11 \\
\hline rs2930593 & 15 & 25877815 & $C$ & A & 18.764 & 2.825 & 44.11 & 4.56E-11 \\
\hline rs12654591 & 5 & 38271582 & C & A & 13.063 & 2.075 & 39.63 & 4.34E-10 \\
\hline rs 2741023 & 2 & 234516714 & G & A & 2.446 & 0.399 & 37.67 & 1.17E-09 \\
\hline rs10179094 & 2 & 234597825 & $\mathrm{~T}$ & A & 2.347 & 0.386 & 37.06 & 1.58E-09 \\
\hline rs7586110 & 2 & 234590527 & $\mathrm{~T}$ & G & 2.344 & 0.385 & 37.05 & 1.59E-09 \\
\hline rs2221198 & 2 & 234658623 & C & $\mathrm{T}$ & 2.251 & 0.374 & 36.33 & 2.29E-09 \\
\hline rs4124874 & 2 & 234665659 & A & C & 2.194 & 0.365 & 36.05 & 2.63E-09 \\
\hline rs3755319 & 2 & 234667582 & $\mathrm{~T}$ & G & 2.194 & 0.365 & 36.05 & 2.63E-09 \\
\hline rs4148326 & 2 & 234673462 & $\mathrm{~T}$ & C & 2.194 & 0.365 & 36.05 & 2.63E-09 \\
\hline rs1876506 & 3 & 42547127 & A & G & 13.734 & 2.308 & 35.43 & 3.61E-09 \\
\hline rs4294999 & 2 & 234635467 & A & G & 2.155 & 0.364 & 35.11 & 4.23E-09 \\
\hline rs2008595 & 2 & 234637192 & G & A & 2.155 & 0.364 & 35.11 & 4.23E-09 \\
\hline rs4663963 & 2 & 234650193 & $\mathrm{~T}$ & G & 2.155 & 0.364 & 35.11 & 4.23E-09 \\
\hline rs16892701 & 8 & 120525983 & G & A & 11.336 & 2.077 & 29.79 & $6.25 \mathrm{E}-08$ \\
\hline rs16892482 & 8 & 120386382 & A & G & 11.326 & 2.077 & 29.74 & $6.41 \mathrm{E}-08$ \\
\hline
\end{tabular}

appeared slightly stronger in the Norfolk Island SNPs than for CEU. Allele frequencies for the 25 SNPs in these 4 populations are detailed in Additional file 4.

Haplotype mapping and association with bilirubin levels Haploview association analysis was performed on the individual 29 SNP 'markers', minor allele frequencies (MAF) and association statistics are documented in Table 3 (for additional information see Additional file 5). All 29 SNPs exhibited significantly $\left(P<1.0 \times 10^{-4}\right)$ increased MAF in the high serum bilirubin group. The most significantly associated marker was rs17863787; the frequency of the ' $G$ ' allele was observed to be $62.3 \%$ in those with high serum bilirubin and $24.9 \%$ in those with normal serum bilirubin $\left(P=5.51 \times 10^{-17}\right)$.

To further investigate the association of genomic structure across the chr2q37.1 region with serum bilirubin, a haplotype association analysis was conducted in Haploview. There were a total of 6 haplotypes inferred for LD block 1 and 7 haplotypes for LD block 2 (Additional file 6); 


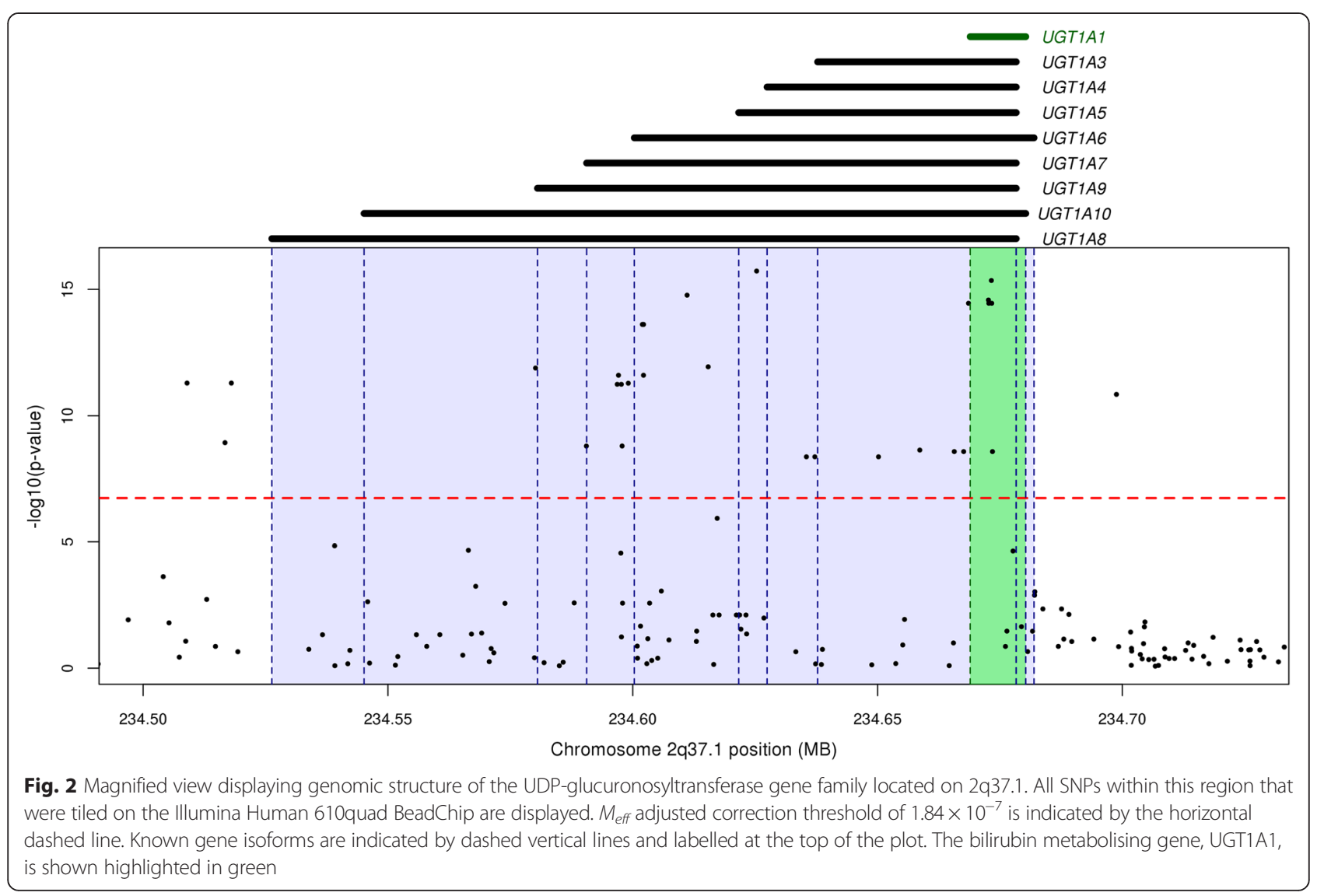

haplotypes present in $>1 \%$ of the total population are shown. The block 1 haplotype most significantly associated with the high bilirubin group was 'TAAGTGGGA', which is estimated to exist at $20.3 \%$ in the total population. This haplotype was observed in $40.3 \%$ of the high serum bilirubin group, and $17.2 \%$ of the normal group $(P=$ $\left.4.59 \times 10^{-9}\right)$. The most abundant block 1 haplotype ('CGGTCCACT', $33.6 \%$ of total population) was observed to be significantly associated with the normal serum bilirubin group; $36.9 \%$ normal vs $19 \%$ high $(P=$ $\left.9.31 \times 10^{-5}\right)$. The LD block 2 haplotype most significantly associated with high serum bilirubin was 'GGGC GTTGTGAGCTTGTTC'; which is estimated to be present in $18.8 \%$ of the total population. This haplotype was observed in $43.5 \%$ of the high serum bilirubin group, and $14.3 \%$ of the normal group $(P=1.73 \times$ $10^{-14}$ ). The most abundant block 2 haplotype ('CAAA TCCACTGTACGTCCT', $49.2 \%$ of total population) was observed to be significantly associated with the normal serum bilirubin group; $54.6 \%$ normal vs $26.1 \%$ high $\left(P=3.51 \times 10^{-9}\right)$. Frequency and combination of the block specific haplotypes is illustrated in Fig. 4.

Nine tagging SNPs were identified that capture the allelic variance of the 29 SNPs (Table 4); the tagging analysis captured all 29 alleles at $r^{2}>=0.8$ which contains
$100 \%$ of alleles with mean $r^{2}$ of 0.963 . These SNPs could be used in future replication analyses to tag variation across the region in other populations.

\section{Bilirubin correlations with clinical metabolic syndrome and cardiovascular disease}

It is well established that serum bilirubin levels are inversely correlated with risk of developing cardiovascular disease [20-22]. Therefore this was investigated using the cardiovascular disease risk score previously calculated for the Norfolk Island population [7], along with potential relationships between other metabolic risk scores, including metabolic syndrome and type- 2 diabetes (scores previously estimated [7]).

A significant inverse relationship was observed between total serum bilirubin and the clinical risk score for metabolic syndrome. Of the 592 individuals with available data $66 \%$ had normal bilirubin levels and no metabolic syndrome, $11.5 \%$ had high bilirubin levels and no metabolic syndrome, $25.3 \%$ had normal bilirubin and metabolic syndrome, $1.2 \%$ had high bilirubin and metabolic syndrome. A chi-squared contingency test followed by Fisher's exact showed that this was a significant observation; $\chi 2=4.18(P=0.04)$, Fisher's Exact OR = $2.45(P=0.03)$. This correlation suggests that Norfolk 


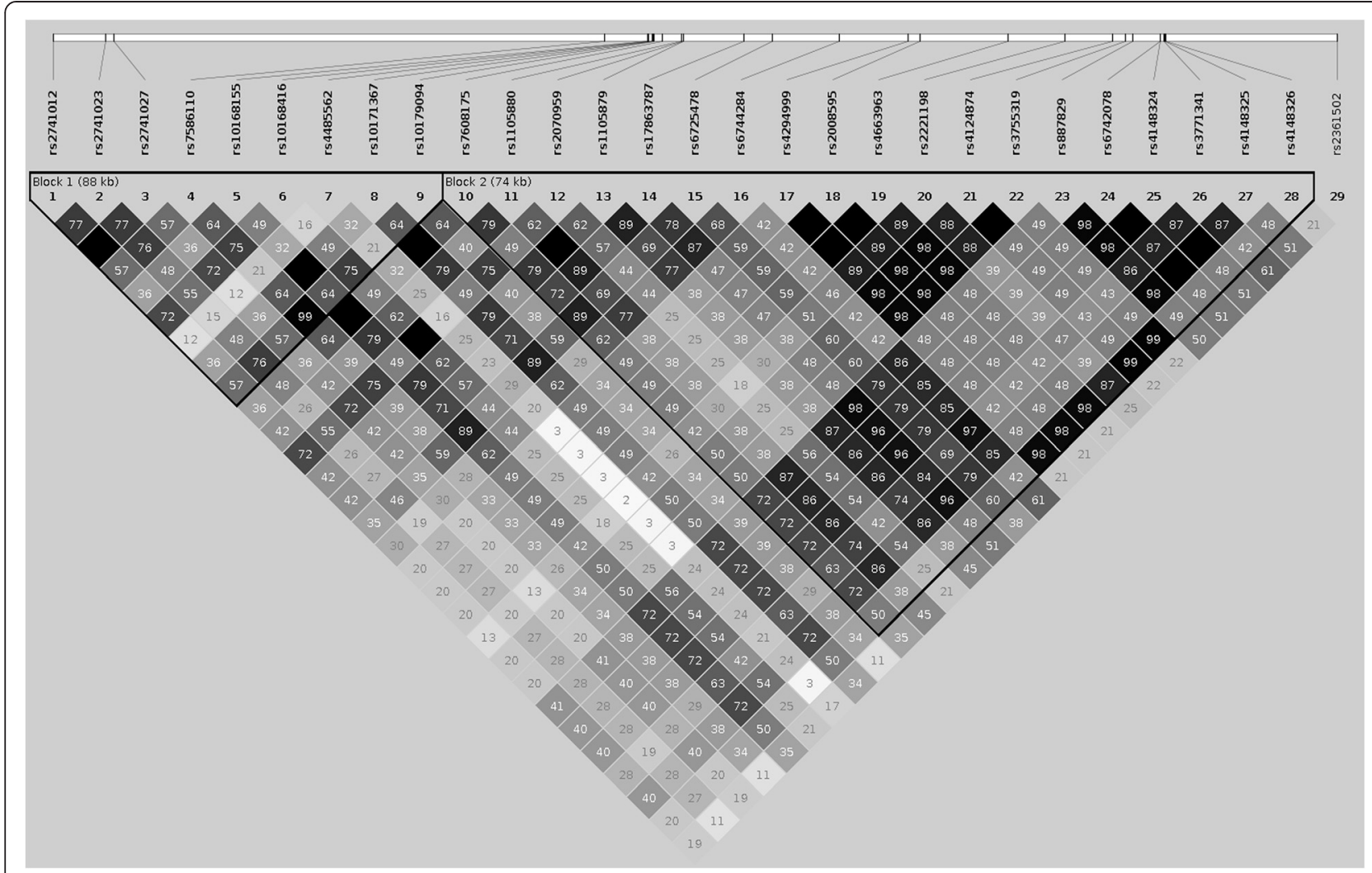

Fig. 3 Linkage Disequilibrium plots for 29 SNPs contained within UDP-glucuronosyltransferase gene family. The 2 LD blocks are outlined in black; Block 1 spans SNPs 1-9, Block 2 spans SNPs 10-28. All SNP rs numbers are listed, with their chromosomal positioning relative to each other indicated at the top of the figure

Island individuals with higher serum bilirubin levels are less likely to develop metabolic syndrome.

Numerous studies have also attributed smoking behaviour to be associated with serum bilirubin levels [23-25]. This was tested in the Norfolk Island population using the students independent $t$-test, and revealed a significant difference in mean serum bilirubin levels between smokers $(6.46 \mu \mathrm{mol} / \mathrm{L})$ and non-smokers $(8.12 \mu \mathrm{mol} / \mathrm{L})$; $\mathrm{t}=3.99$ with $\mathrm{P}=4.06 \times 10^{-5}$.

To further examine potential relationships a series of t-tests between a variety of quantitative metabolic syndrome/cardiovascular disease traits and categorised serum bilirubin group were performed. There were a total of 9 significant $(P<0.05)$ trait correlations with categorised bilirubin level, these were; body mass index (BMI), body fat, cholesterol/HDL-C ratio, total cholesterol, hip circumference, LDL-C, type-2 diabetes risk score, total protein and triglycerides (Table 5). These findings highlight traits that are consistent with previous literature $[26,27]$.

Body fat was observed to have the strongest correlation with serum bilirubin, with significantly reduced body fat composition in individuals who had high serum bilirubin levels. Unlike previous observations [20, 27, 28], cardiovascular disease risk score was not significantly reduced in those individuals with higher serum bilirubin, whereas, type- 2 diabetes risk did show a significant reduction in the higher bilirubin group, consistent with previous literature $[26,29]$.

\section{Genotype effects on metabolic syndrome, type-2 diabetes and cardiovascular disease traits}

To further explore the above approach, associations between the 29 significantly associated SNPs and metabolic traits other than serum bilirubin were explored. Traits which showed a significant $(\mathrm{P}<0.05)$ correlation with total serum bilirubin (Table 5) were selected. Only one trait was observed which showed a significant association with any of the 29 markers, this was type-2 diabetes-risk when categorised: "low"; "intermediate", and "high" [9]. Using a chi-squared test rs2741012 and rs2741027 were significantly associated with type- 2 diabetes-risk $(x 2=9.63, P=$ 0.0069). Again this was followed with a Fisher's Exact test which confirmed significance $(P=0.0081)$. The same observation with the minor allele and suggestive protection was observed.

To further investigate the above associations logistic regression was used to identify a model that predicts outcome 
Table 3 Haploview marker associations showing frequencies of the recessive alleles

\begin{tabular}{|c|c|c|c|c|c|c|}
\hline SNP & Associated allele & High bili allele freq & Normal bili allele freq & OR $(95 \%$ Cl) & Chi square & $P$ value \\
\hline rs2741012 & $\mathrm{T}$ & 0.415 & 0.188 & $0.33(0.22-0.49)$ & 31.93 & $1.60 \mathrm{E}-08$ \\
\hline rs 2741023 & A & 0.431 & 0.239 & $0.41(0.28-0.61)$ & 20.06 & 7.52E-06 \\
\hline rs 2741027 & A & 0.415 & 0.188 & $0.33(0.22-0.49)$ & 31.93 & $1.60 \mathrm{E}-08$ \\
\hline rs7586110 & G & 0.500 & 0.251 & $0.33(0.23-0.49)$ & 32.33 & 1.30E-08 \\
\hline rs10168155 & $\mathrm{T}$ & 0.654 & 0.326 & $0.26(0.17-0.38)$ & 48.96 & 2.62E-12 \\
\hline rs10168416 & G & 0.492 & 0.194 & $0.25(0.17-0.37)$ & 51.93 & $5.75 \mathrm{E}-13$ \\
\hline rs4485562 & G & 0.800 & 0.626 & $0.42(0.26-0.66)$ & 14.44 & 1.00E-04 \\
\hline rs10171367 & G & 0.654 & 0.326 & $0.26(0.17-0.38)$ & 48.96 & $2.62 \mathrm{E}-12$ \\
\hline rs10179094 & A & 0.500 & 0.249 & $0.33(0.22-0.49)$ & 32.83 & $1.01 \mathrm{E}-08$ \\
\hline rs7608175 & G & 0.654 & 0.326 & $0.26(0.17-0.38)$ & 49.11 & $2.42 \mathrm{E}-12$ \\
\hline rs1105880 & $C$ & 0.646 & 0.271 & $0.20(0.14-0.30)$ & 68.04 & 1.60E-16 \\
\hline rs2070959 & G & 0.492 & 0.194 & $0.25(0.17-0.37)$ & 51.93 & $5.75 \mathrm{E}-13$ \\
\hline rs1105879 & G & 0.646 & 0.271 & $0.20(0.14-0.30)$ & 68.04 & $1.60 \mathrm{E}-16$ \\
\hline rs17863787 & G & 0.623 & 0.249 & $0.20(0.13-0.30)$ & 70.15 & $5.51 \mathrm{E}-17$ \\
\hline rs6725478 & $\mathrm{T}$ & 0.631 & 0.300 & $0.25(0.17-0.37)$ & 51.58 & $6.88 \mathrm{E}-13$ \\
\hline rs6744284 & $\mathrm{T}$ & 0.577 & 0.222 & $0.21(0.14-0.31)$ & 66.86 & $2.91 \mathrm{E}-16$ \\
\hline rs4294999 & G & 0.715 & 0.429 & $0.30(0.21-0.45)$ & 35.46 & 2.61E-09 \\
\hline rs2008595 & A & 0.715 & 0.429 & $0.30(0.21-0.45)$ & 35.46 & 2.61E-09 \\
\hline rs4663963 & G & 0.715 & 0.429 & $0.30(0.21-0.45)$ & 35.46 & 2.61E-09 \\
\hline rs2221198 & $\mathrm{T}$ & 0.672 & 0.402 & $0.33(0.22-0.49)$ & 31.32 & 2.18E-08 \\
\hline rs4124874 & C & 0.715 & 0.427 & $0.30(0.21-0.45)$ & 35.86 & 2.12E-09 \\
\hline rs3755319 & G & 0.715 & 0.427 & $0.30(0.21-0.45)$ & 35.86 & 2.12E-09 \\
\hline rs887829 & A & 0.623 & 0.254 & $0.20(0.14-0.30)$ & 67.92 & $1.70 \mathrm{E}-16$ \\
\hline rs6742078 & $\mathrm{T}$ & 0.623 & 0.253 & $0.20(0.14-0.30)$ & 68.23 & $1.46 \mathrm{E}-16$ \\
\hline rs4148324 & G & 0.623 & 0.254 & $0.20(0.14-0.30)$ & 67.92 & $1.70 \mathrm{E}-16$ \\
\hline rs3771341 & $\mathrm{T}$ & 0.577 & 0.227 & $0.22(0.15-0.32)$ & 64.55 & $9.40 \mathrm{E}-16$ \\
\hline rs4148325 & $\mathrm{T}$ & 0.623 & 0.254 & $0.20(0.14-0.30)$ & 67.92 & 1.70E-16 \\
\hline rs4148326 & C & 0.715 & 0.427 & $0.30(0.21-0.45)$ & 35.86 & 2.12E-09 \\
\hline rs2361502 & $C$ & 0.546 & 0.268 & $0.30(0.21-0.45)$ & 38.61 & 5.16E-10 \\
\hline
\end{tabular}

Note: odds ratios are not adjusted for age and sex

(type-2 diabetes) from trait (bilirubin) and factors in potential modifiers (genotype). Logistic regression modelling identified direct bilirubin as being significantly associated with categorised type- 2 diabetes risk $\left(\mathrm{r}^{2}: 0.05, p\right.$-value: $0.005)$, suggesting that in the Norfolk Island cohort increased direct bilirubin was associated with a $28 \%$ reduction in type-2 diabetes risk (OR:0.72, 95 \% CI: 0.57-0.91). Based on a bi-directional stepwise regression model approach 2 of the 9 tagging SNPs remained significant; rs2741027 and rs6725478. These SNPs effectively tag the two major 'protective'/high bilirubin haplotypes. When included, the adjusted model remained significant $\left(r^{2}: 0.13\right.$, p-value: 0.0001) and confirmed the initial association; direct bilirubin (OR:0.70, 95 \% CI: 0.53-0.89, p-value: 0.005): rs2741027 (OR:0.25, 95 \% CI: 0.10-0.58, p-value: 0.002), rs6725478 (OR:0.27, 95 \% CI: 0.10-0.63, p-value: 0.004).
This indicates that when controlling for bilirubin levels genotype affects risk of type-2 diabetes within the Norfolk Island population. Therefore, inclusion of SNP genotypes when assessing the relationship between direct bilirubin and type- 2 diabetes risk increases the accuracy of the 'risk' estimate within the Norfolk Island cohort.

Functional Annotation of UDP-glucuronosyltransferase SNPs Investigation of the 29 SNPs revealed several of potential functional interest (SNP annotation Table 6). Three SNPs are within the coding region of UGT1A6 (Table 6); rs1105880 (synonymous), rs1105879 and rs2070959 (non-synonymous). Further investigation with SNPnexus (http://www.snp-nexus.org/) revealed rs1105879 had a PolyPhen score of 'possibly damaging', indicating the usually conserved nature of the coded amino acid. Six 


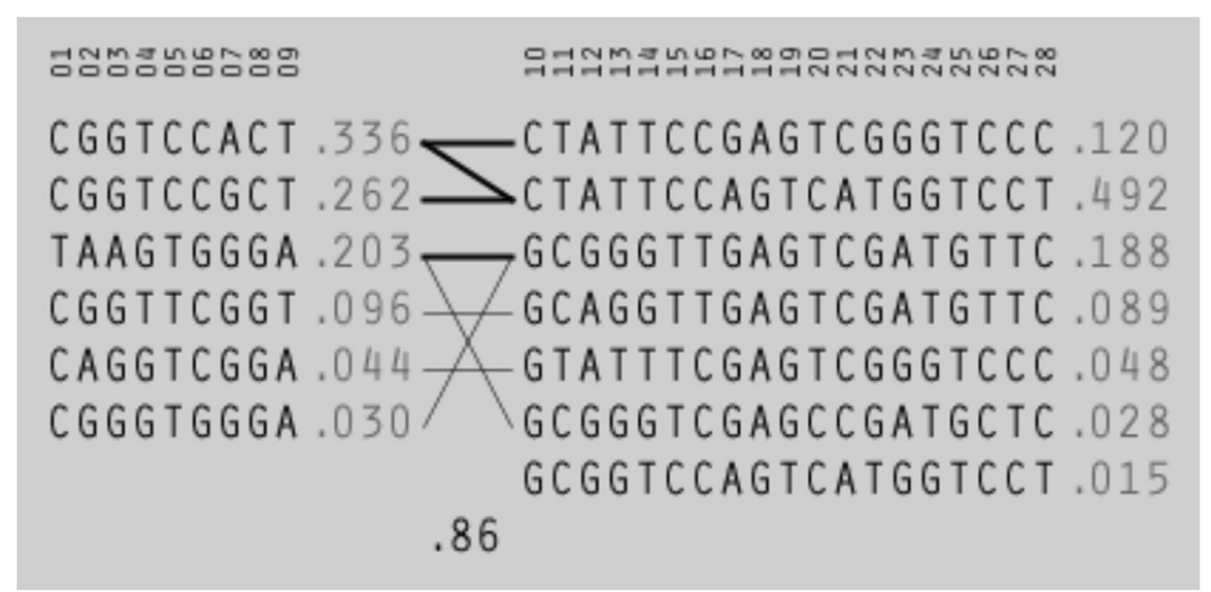

Fig. 4 Haplotype structure across the two identified LD blocks in the Norfolk Island cohort UDP-glucuronosyltransferase gene family. Displayed haplotypes reside at $>1 \%$ frequency in the genotyped samples. Connecting lines represent haplotype combinations: thick lines represent haplotype combinations that reside at $>10 \%$, thin lines $>1 \%$ of samples

SNPs were observed to reside within $5^{\prime}$ prime untranslated regions (5'UTR); UGT1A1 (rs887829, rs3755319), UGT1A3 (rs2008589), UGT1A6 (rs7608175), UGT1A7 (rs7586110), and UGT1A9 (rs2741045).

\section{Discussion}

We have identified a significant genomic association at $2 q 37.1$ in the region of the UDP-glucuronosyltransferase (UDPGT) enzyme family members, with direct and total serum bilirubin levels. Correlation analyses between metabolic syndrome related traits and serum bilirubin levels identified significant inverse relationships for numerous traits. Haplotype association testing revealed the presence of potentially protective haplotypes within the Norfolk Island population. Thus this study has identified a complex region which shows interplay between genomic and environmental conditions and has a large effect on overall serum bilirubin levels.

Previous literature has suggested a linkage between bilirubin and metabolic risk with clinical associations observed between cardiovascular disease risk, obesity and bilirubin concentrations [20-22, 27] and more recently metabolic syndrome [30-34]. Therefore, we investigated potential relationships between bilirubin and metabolic traits in the Norfolk Island cohort. An inverse correlation between serum bilirubin and several important metabolic traits was observed, with the most notable being metabolic syndrome and type-2 diabetes risk. Given that metabolic syndrome and type- 2 diabetes increase cardiovascular disease risk it is consistent with the current body of literature which documents inverse association between high serum bilirubin and cardiovascular disease risk (review [26]).

Our analysis refined an association with serum bilirubin concentration to a $189.8 \mathrm{~kb}$ region on chromosome $2 q 37.1$ with genotypic analyses revealing that the level of serum bilirubin was greatly increased in individuals with the rare allele. This region encodes one of the major drug metabolising families (UDP-glucuronosyltransferase, UDPGT) [35-37]; there are 9 documented

Table 4 Haploview 'Tagger' analysis of the 29 GWAS associated chr2q37.1 SNPs identified 9 SNPs as tagging the allelic variation across the region

\begin{tabular}{|c|c|}
\hline SNP tested & Alleles captured \\
\hline rs4148325 & rs3771341,rs887829,rs1 105879,rs4148325,rs6742078,rs17863787,rs1 105880,rs6744284,rs4148324 \\
\hline rs2008595 & rs4294999,rs4148326,rs4663963,rs2221 198,rs3755319,rs4124874,rs2008595 \\
\hline rs6725478 & rs6725478,rs10168155,rs10171367,rs7608175 \\
\hline rs7586110 & rs10179094,rs7586110 \\
\hline rs10168416 & rs10168416,rs2070959 \\
\hline rs 2741027 & rs2741027,rs2741012 \\
\hline rs 2741023 & rs2741023 \\
\hline rs2361502 & rs2361502 \\
\hline rs4485562 & rs4485562 \\
\hline
\end{tabular}


Table 5 Metabolic trait correlation with serum bilirubin group

\begin{tabular}{|c|c|c|c|c|}
\hline Trait & $\mathrm{t}$ & $P$ & High bili mean & Normal bili mean \\
\hline albumin & 0.90 & 3.70E-01 & 41.75 & 41.43 \\
\hline Body Mass Index* & -1.91 & $3.00 \mathrm{E}-02$ & 25.06 & 26.27 \\
\hline body fat* & -3.43 & 5.30E-04 & 26.63 & 30.67 \\
\hline cholesterol/HDL-C ratio* & -1.82 & 4.00E-02 & 3.94 & 4.29 \\
\hline cholesterol* & -2.71 & 4.00E-03 & 5.30 & 5.67 \\
\hline creatinine & 0.15 & 8.80E-01 & 80.19 & 79.90 \\
\hline cardiovascular disease risk & 0.40 & $6.90 \mathrm{E}-01$ & 7.20 & 6.69 \\
\hline diastolic blood pressure & 0.51 & $6.90 \mathrm{E}-01$ & 77.69 & 76.68 \\
\hline globin & 1.91 & $6.10 \mathrm{E}-02$ & 30.29 & 29.34 \\
\hline $\mathrm{HDL}-\mathrm{C}$ & 0.62 & 5.30E-01 & 1.44 & 1.41 \\
\hline hip circumference* & -2.04 & $2.00 \mathrm{E}-02$ & 99.42 & 101.92 \\
\hline Inbreeding & -0.42 & 3.40E-01 & 0.01 & 0.01 \\
\hline LDL-C ${ }^{*}$ & -1.66 & 5.00E-02 & 2.66 & 2.86 \\
\hline mean arterial pressure & 0.26 & 8.00E-01 & 95.01 & 94.44 \\
\hline Polynesian Admixture & -1.41 & 8.50E-02 & 0.21 & 0.25 \\
\hline pulse pressure & 0.02 & $9.80 \mathrm{E}-01$ & 52.31 & 52.26 \\
\hline systolic blood pressure & 0.29 & $6.10 \mathrm{E}-01$ & 130.00 & 128.95 \\
\hline type-2 diabetes risk* & -2.31 & $1.00 \mathrm{E}-02$ & 9.19 & 11.22 \\
\hline total protein* & 2.28 & 3.00E-02 & 72.10 & 70.79 \\
\hline triglycerides* & -2.15 & 2.00E-02 & 1.58 & 2.00 \\
\hline urea & -0.44 & $6.60 \mathrm{E}-01$ & 5.49 & 5.58 \\
\hline uric acid & 0.53 & $6.00 \mathrm{E}-01$ & 0.35 & 0.34 \\
\hline waist circumference & -1.91 & 1.30E-01 & 85.04 & 87.62 \\
\hline waist-hip ratio & 0.34 & $6.30 \mathrm{E}-01$ & 0.86 & 0.86 \\
\hline weight & 0.20 & 5.80E-01 & 75.79 & 75.31 \\
\hline
\end{tabular}

* significant at $P<0.05$

UDPGT isoforms; UGT1A1, UGT1A3, UGT1A4, UGT 1A5, UGT1A6, UGT1A7, UGT1A8, UGT1A9 and UG T1A10 (Fig. 2). UGT1A1 is well known to preferentially metabolise bilirubin and has been previously mapped in linkage and GWAS studies [16-18, 38-43]. UGT1A3 and UGT1A4 also have been shown to have potential action with bilirubin [37]. However all gene family members, including UGT1A1, exhibit affinity for numerous substrates and it is therefore possible that the gene effects are not mediated (entirely) by total bilirubin. Such pleotropic effects at this loci are likely to be the case as evidenced by the fact that adjustment for

Table 6 Functional annotation of the chr2q37.1 SNPs significantly associated with total serum bilirubin levels

\begin{tabular}{|c|c|c|c|c|c|}
\hline$\overline{R e f S N P ~ I D ~}$ & NCBI gene ID & Gene symbol & Function class & Residue & Amino acid position \\
\hline rs1105879 & 54578 & UGT1A6 & coding-nonsynonymous & $\mathrm{S}$ & 183 \\
\hline rs2070959 & 54578 & UGT1A6 & coding-nonsynonymous & A & 180 \\
\hline rs1105880 & 54578 & UGT1A6 & coding-synonymous & L & 104 \\
\hline rs2008595 & 54659 & UGT1A3 & $5^{\prime} \mathrm{UTR}$ & - & - \\
\hline rs2741045 & 54600 & UGT1A9 & $5^{\prime}$ UTR & - & - \\
\hline rs3755319 & 54658 & UGT1A1 & 5'UTR & - & - \\
\hline rs7586110 & 54577 & UGT1A7 & $5^{\prime}$ UTR & - & - \\
\hline rs7608175 & 54578 & UGT1A6 & 5'UTR & - & - \\
\hline rs887829 & 54658 & UGT1A1 & 5'UTR & - & - \\
\hline
\end{tabular}


serum bilirubin in our modelling did not completely nullify the observed association between genotype and outcome. Future work is required to more fully explore these effects along with associations of other substrates with variants at this genomic region.

Mutations in UGT1A1 have also been associated with Crigler-Najjar syndromes types I and II and in Gilbert syndrome [44-46]. Gilbert Syndrome (GS) is a welldocumented benign increase in serum bilirubin, and is caused by the reduced activity of UDPGT [47-51]. In line with the observations that serum bilirubin is inversely correlated with metabolic risk diabetic patients with GS are less likely to develop vascular dysfunctions [52]. Furthermore, the incidence of diabetes and cardiovascular disease risk mortality is lower in GS individuals, with one study exploring the efficacy of increasing serum bilirubin in type-2 diabetic patients [53]. Further evidence confirming the protective role of circulating bilirubin for type- 2 diabetes has been reported in a prospective study [19].

Significant difference has been identified between functional polymorphisms within the UGT1A family between Caucasian and other populations [54]. Polymorphisms in the promoter region for UGT1A1 (2 bp TA insertion in the TATA box) increased activity in Caucasian GS patients; this was not observed in Asian and African GS patients or Pacific populations [54]. The authors suggest that due to the complex nature of environmental and genetic factors, unstable polymorphisms within UGT1A1 may act to "fine-tune" plasma bilirubin levels on a population by population basis, meaning that the promoter variation explains the presence of GS in some populations, but in other populations it's more likely a combination of variants in the encoding region along with environmental factors [54], our data supports this hypothesis. Additionally, metaanalysis has demonstrated strong replication for a genetic influence on serum bilirubin levels of the UGT1A1 locus $\left(P<5 \times 10^{-324}\right)$, specifically at the proximal promoter region of UGT1A1 tagged by rs6742078 [40]. While we didn't have genotype information for this SNP we were able to impute against the 1000 Genomes panel to extrapolate associations between the two studies. Using imputed information we were able to illustrate that there is tight LD between rs6742078 and the top associated SNP from our study, rs6744284 $\left(r^{2}=0.85\right)$, suggesting that the Norfolk Island cohort exhibit a similar genetic pattern of association.

We identified strong LD across the region of 2q37.1, potentially suggesting that the Norfolk Island population's unique genomic structure is influencing serum bilirubin concentration. LD across the same region in data available through the HapMap project [55] showed that the Norfolk Island cohort exhibited an LD pattern similar to that observed in the European population (CEU), while both the Asian populations (Chinese and Japanese) exhibited very different genetic structure across this region. This is not unexpected because of the large amount of recent European admixture in the Norfolk population. Additionally, it was noted that haplotypes containing the minor allele(s) in the Norfolk Island population potentially conferred protection to metabolic disorders as measured by clinical metabolic syndrome and type- 2 diabetes-risk. It is possible that selection is driving the presence of high serum bilirubin within populations, although this may be achieved by different variation across the region. It appears that in Europeans this variation is often in the promoter region, whereas in Asian and African populations this is not the case, and it is polymorphisms in the gene body that seems to account for the associations with increased bilirubin. This strongly suggests that it is beneficial for a population to have a certain frequency of individuals with naturally high serum bilirubin, and potentially points to a complex interaction between environmental and genomic factors maintaining this.

One significant association between 2 SNPs (rs2741012 and rs2741027) and categorised type-2 diabetes-risk was observed. These two SNPs are just upstream of the promoter and 5'UTR region of the UDPGT family. It is likely that these SNPs are in LD with untyped polymorphisms (SNPs not on the 610quad chip) that reside in these regions and potentially form a LD block/haplotype in the Norfolk Island population which confers protection to type-2 diabetes as well as metabolic syndrome. Interestingly, and in support of our approach, this reduction in risk correlates well with previous work conducted in a large US cohort [13]; these variants (or variants tagged by them) may be functional, i.e. they might directly affect transcription and/or translation of the isoforms encoded by the UDPGT family. It is also possible that there are additional rare variants within the region that further influence serum bilirubin as recently evidenced by an exome sequencing study performed in elderly individuals [56].

Given that bilirubin is a cheap and commonly measured laboratory test, routine screening of serum bilirubin levels could be beneficial in the stratification and treatment of metabolic disorders such as cardiovascular disease and type- 2 diabetes. Identification of genes/variants that exhibit pleiotropic effects (effects of the same variant on multiple characteristics or disease risks) is an ultimate goal. The significant interaction observed here provides evidence that bilirubin may be affected by genetic and environmental factors and their interactions.

\section{Conclusions}

In summary, this study identified strong associations of variants within the UGT1A family with regulation of serum bilirubin levels in the Norfolk Island population, which replicated previous GWAS and epidemiological 
findings. This successful implementation of pedigree-based analysis using the unique properties of the Norfolk Island cohort highlights a functional region that offers protective benefit from metabolic disease and further eludes to a potentially heritable component with the Norfolk Island population. Specific haplotype structure was significantly associated with increased serum bilirubin, and as such this study has identified a potential set of 'protective' haplotypes that exist within the Norfolk Island population. Further studies are warranted to validate these findings, with the next step being to explore these associations in larger outbred populations.

\section{Methods}

Sample/cohort collection, pedigree information and ethics The Norfolk Island Health Study (NIHS) is well established with regards to data collection and initial disease prevalence studies $[4,5,8]$. The Norfolk Island pedigree structure has been previously outlined [57], and subsequently updated [1]. The most recent update led to the reconstruction of a core-pedigree consisting of 1388 members coalescing over 11 generations (or 200 years) back to the original founders. [3, 7]. This study focuses on a reduced corepedigree, meaning that individuals; a) are genetically related to the original founders, and b) have phenotype and genotype information available. The total number of individuals fitting these criteria was 382. All individuals gave written informed consent. Ethical approval was granted prior to the commencement of the study by the Griffith University Human Research Ethics Committee (ethical approval no: 1300000485 ) and the project was carried out in accordance with the relevant guidelines, which complied with the Helsinki Declaration for human research.

\section{Heritability analysis}

All 19 metabolic traits assessed in this analysis are part of the NIHS2000 collection [8]. Traits measured were: fasting plasma glucose, HDL-C, LDL-C, total plasma cholesterol, cholesterol-HDL-C ratio, triglycerides, creatinine, total protein, globin, albumin, urea, uric acid, total serum bilirubin, direct serum bilirubin, and numerous enzymes that are markers for liver/kidney function (ALT, AST, Alkaline Phosphatase, GGT, Lactate Dehydrogenase [LDH]).

\section{Heritability estimates}

The $\mathrm{R}$ package and genetic analysis program GenABEL [58] was used to calculate heritability estimates for all metabolic syndrome/cardiovascular disease related traits. The genetic kinship matrix derived from the SNP data and reconstructed core-pedigree was used to estimate trait heritability $\left(\mathrm{h}^{2}\right.$ [narrow-sense heritability]) by polygenic modelling. All traits were screened for covariant effects of age and sex interactions.

\section{Genome-wide SNP genotyping}

EDTA anticoagulated venous blood samples were collected from all participants. Genomic DNA was extracted from blood buffy coats using standard phenol-chloroform procedures. DNA samples were genotyped according to the manufacturer's instructions on Illumina Infinium High Density (HD) Human610-Quad DNA analysis BeadChip version 1. BeadChips were a four-sample format requiring $200 \mathrm{ng}$ of DNA per sample. Samples were scanned on the Illumina BeadArray 500GX Reader. Raw data was obtained using Illumina BeadScan image data acquisition software (version 2.3.0.13). Raw data from Illumina idat files were SNP genotyped in $\mathrm{R}$ using the CRLMM package [59]. Genotype data then underwent initial quality control routines using PLINK [60]. SNPs were filtered based on: minor allele frequency $>0.01$; call rate $>0.95$, and HardyWeinberg equilibrium testing p-value $>10^{-5}$. After this initial quality control, 590,603 SNPs were exported from PLINK and imported into the CRAN package GenABEL [58]. Further filtering (including Mendelian inheritance violations and sex-checking based on available $\mathrm{X}$ and $\mathrm{Y}$ markers) in GenABEL lead to the reduction of the SNP set to a total of $\sim 480,000$; this included removal of both $X$ and $\mathrm{Y}$ chromosome SNPs after gender checking, as well as the removal of mitochondrial and XY SNPs.

\section{Genome-wide association analysis}

A pedigree based GWAS analysis of all heritable traits was batched using custom $\mathrm{R}$ scripts and the package GenABEL [58]. GenABEL uses an additive approach and the loci are coded as $0,1,2$ (corresponding to genotypes AA, $\mathrm{AB}$, and $\mathrm{BB}$, respectively). A detailed explanation of the association model and specific GWAS overview as implemented in the Norfolk Island was previously described [7]. Breifly, a correction was made for the relatedness inherent in the Norfolk Island population using the polygenic model with age and sex interactions, as well as genetic structure [the top 2 genomic principal components of the complete SNP set as calculated by KING [61]]. The top two components were chosen as covariates because we found that these explained the majority of the variance in the outcomes being tested and because inclusion of additional, less informative components only served to reduce the parsimony of the models. For association analysis the mmscore function implemented in GenABEL was used. This function represents a mixed model approximation analysis for association between a trait and genetic polymorphism(s), and is specifically designed for association testing in samples of related individuals. This allows for per SNP association testing using a mixed model polygenic approach. After correcting for multiple testing, the study-wide significance was set based on $M_{\text {eff }}$ adjustment $\left(\mathrm{P}=1.84 \times 10^{-7}\right)$. It should be noted that this $M_{\text {eff }}$ threshold is tailored to trait-wise associations, not multi-trait 
analyses therefore p-values are adjusted on a per trait basis. Association statistics for every SNP for each trait were generated and output to compressed files (.gz.tar) for storage and future reference. GWAS Manhattan plots where generated for each trait association using a custom modified version of the GenABEL plot.scan.gwaa function (for all Manhattan plots see Additional file 2). Annotation of the robustly associated bilirubin SNPs identified as being functional was performed using: http://brainarray.mbni.med.umich.edu/Brainarray/Database/SearchSNP/snpfunc.aspx.

\section{LD testing and haplotype association}

Genotype data for the chrq37.12 region was phased using SHAPEIT2 [62], which has functionality to deal with complex pedigree structures - implemented through the duoHMM algorithm. From this process we observed no Mendelian errors before moving the phased data over to Haploview analyses. Haplotype/LD testing, SNP tagging and association analyses were all conducted in Haploview 4.2 [63]. LD blocks were determined using the default Haploview settings which infer LD based on a pairwise comparison of correlation $\left(\mathrm{r}^{2}\right)$ values between SNPs. Haplotypes were inferred from the genotypes of SNPs which made up the identified LD blocks, and were only recorded if they existed in more than $1 \%$ of the population. Tagging SNPs were determined using the 'tagger' option of Haploview, using a pair-wise tagging method with a minimum observed $r^{2}$ between pairs of 0.8 . Association analyses were carried out on both markers (SNPs) and haplotypes using the inbuilt Haploview association function. A phenotype column was added to the dataset to allow a 'case'/'control' experimental set-up; where case represented the high bilirubin group and control the normal bilirubin group. There were a total of 65 cases and 317 controls with 124 genotyped individuals missing phenotype information. Permutation testing was run to confirm the above association analyses for both marker and haplotype associations. To ensure the robustness of final $\mathrm{P}$ values the number of permutations was set at 1,000,000 (this should lead to a reduction of the FDR). A further exploration of potentially similar structure across the region spanned by the 29 SNPs was tested in 3 HapMap populations; CEU (European), CHD (Chinese), and JPT (Japanese). Due to data being generated on different genotype platforms (SNP chips), a final list of 25 consensus SNPs was retained for Haploview analysis for Norfolk Island and the 3 HapMap sets. Linkage disequilibrium plots across the 25 SNP region was generated for each of the 4 populations (Additional file 3).

\section{Correlations with metabolic traits}

Initial exploratory correlations between risk scores for cardiovascular disease and type- 2 diabetes, clinically defined Metabolic Syndrome (categorical: 0 (no MetS), 1 (MetS)), and various related traits were conducted in R 2.15.2 [64].
For all analyses total serum bilirubin levels were categorised into 'normal' and 'high' groupings, with 'high' being defined as $>14 \mu \mathrm{mol} / \mathrm{L}$, this approximates a clinical cut-off and allows facilitates interpretation in line with existing clinical guidlines. For all other traits tested a standard student's $t$-test (as implemented in R) was used to test for a significant difference of means between the given trait and bilirubin level. There were two categorical traits tested for correlation with serum bilirubin levels; smoking and presence of metabolic syndrome. Smoking has been previously well documented to be associated with serum bilirubin levels [23], and was categorised in the Norfolk Island cohort as either 'yes' (smokers $N=133$ ) or 'no' (non-smokers $N=458$ ). Correlation testing between smoking and bilirubin was carried out using a $2 \times 2$ chi-squared contingency test, followed by a Fisher's Exact test. For correlation analysis between total serum bilirubin and metabolic syndrome there were a total of 598 individuals with available matched phenotype data; 'metabolic syndrome' $(N=156)$ and 'no metabolic syndrome' $(N=442)$. The clinical diagnosis of metabolic syndrome previously calculated for the Norfolk Island cohort was used [7]. A $2 \times 2$ chi-squared contingency test was used to evaluate the significance, followed by a Fisher's Exact test as one of the tables cells contained a value less than $5 \%$. Due to the initial exploratory nature of these analyses all tests are unadjusted, so nominal $\mathrm{p}$-values are reported. Additionally, relatedness within the population is accounted for in later formal modelling using GLM regression.

\section{Regression modelling testing association between outcome, trait and genotype}

To further explore associations between bilirubin, type- 2 diabetes and the genotypic architecture across the UGT1A1 region regression modelling was conducted in $\mathrm{R}$. To establish an initial association, separate logistic regression was conducted between categorised type- 2 diabetes risk and total bilirubin and then direct bilirubin. Additionally a bidirectional stepwise logistic regression model was used to test the significance of each of the 9 tagging SNPs identified in the LD block analysis. The model was not corrected for common covariates (age, sex, smoking, BMI) as these are all accounted for in the calculation of the AUSDRISK type2 diabetes risk score (as previously calculated in the Norfolk Island cohort [7]). To address the issue of relatedness we included the average pedigree kinship as a covariate in the stepwise regression model. This was excluded from the final model indicating that in this instance relatedness is not a significant issue. Reported $r^{2}$ values use the Nagelkerke Index pseudo $r^{2}$ as calculated in $R$. Model $p$ values were generated from an ANOVA using the F distribution, which tests the null hypothesis that the coefficients represented in the overall regression model (represented by $\mathrm{R}$ 2) are equal to 0. 


\section{Web resources}

Online Mendelian Inheritance in Man (http://www.omim.org)

Catalogue of genome-wide association studies (http:// www.genome.gov/gwastudies)

BrainArray (http://brainarray.mbni.med.umich.edu/Brainarray/Database/SearchSNP/snpfunc.aspx).

\section{Availability of supporting data}

Due to current ethical constraints, restricted data access is in place to anonymise genotypic SNP GWAS and phenotype data. The Norfolk Island Health Study steering committee will assess restricted data access requests via our GRC computational genetics group (interested researchers should contact grccomputationalgenomics@gmail.com).

\section{Additional files}

Additional file 1: Summary statistics of phenotypic traits measured in the Norfolk Island population. Table with an overview of the Norfolk Island phenotype data analysed, 16 traits in total. (PDF $96 \mathrm{~kb}$ )

Additional file 2: GWAS Manhattan plots for metabolic related traits. GWAS Manhattan plots for all 16 traits. (PDF $7896 \mathrm{~kb}$ )

Additional file 3: LD lots for $\mathbf{4}$ populations across $200 \mathrm{~kb}$ of chr2q37.1. Haploview LD plots for 25 SNPs spanning a region of chr2q37.1 for four populations; NI (Norfolk Island), CEU (European); CHD (Chinese), and JPT (Japanese). (PDF 2809 kb)

Additional file 4: Haploview allele frequency data for 25 chr2q37.1 SNPs across 4 populations. Minor allele frequencies for 25 SNPs across five populations; Norfolk Island (NI), European (CEU), Chinese (CHD and CHB), and Japanese (JPT). (PDF $137 \mathrm{~kb}$ )

Additional file 5: Detailed Haploview allele frequency data for all 29 SNPs in the NI cohort. Allele frequency data for all 29 SNPs across the chr2q37.1 region for the Norfolk Island samples. (PDF $155 \mathrm{~kb}$ )

Additional file 6: Haploview haplotype associations with bilirubin levels. Haplotypes identified by Haploview and their association statistics. (PDF $33 \mathrm{~kb}$ )

\section{Abbreviations}

BMI: Body mass index; CEU: European Hapmap population; CHD: Chinese Hapmap population; CVD: Cardiovascular disease; DBP: Diastolic blood pressure; GS: Gilbert's syndrome; GWAS: Genome-wide association study; $h^{2}$ : Heritability (narrow-sense); HDL-C: High-density lipoprotein cholesterol; JPT: Japanese Hapmap population; LD: Linkage disequilibrium; LDL-C: Low-density lipoprotein cholesterol; MAF: Minor allele frequency; Nl: Norfolk Island; NIHS: Norfolk Island health study; SBP: Systolic blood pressure; SNP: Single nucleotide polymorphism.

\section{Competing interests}

The authors declare that they have no competing interests.

\section{Authors' contributions}

$C B, M C$, and $J C$ carried out the genotype assays. MH curated phenotype and pedigree data. $\mathrm{MB}, \mathrm{RL}$, and $\mathrm{DE}$ participated in the design of the study and performed the statistical analysis. JB provided detailed statistical expertise and critical evaluation of methodology. MB, RL and LG conceived of the study, and participated in its design. DM and GC helped to draft the manuscript, provided critical revision and intellectual input into the final manuscript design. All authors read and approved the final manuscript.

\section{Acknowledgements}

This research was supported by funding from a National Health and Medical Research Council of Australia (NHMRC) Project Grant. It was also supported by infrastructure purchased with Australian Government ElF Super Science Funds as part of the Therapeutic Innovation Australia - Queensland Node project. Also Miles Benton was supported by a Corbett Postgraduate Research Scholarship. We would like to acknowledge Amanda Miotto and also QUT for providing computational support for this project. Lastly, we extend our appreciation to the Norfolk Islanders who volunteered for this study.

\section{Author details}

'Genomics Research Centre, Institute of Health and Biomedical Innovation, Queensland University of Technology, Kelvin Grove, QLD 4059, Australia. ${ }^{2}$ Kenepuru Science Centre, Institute of Environmental Science and Research, Wellington 5240, New Zealand. ${ }^{3}$ Texas Biomedical Research Institute, San Antonio, TX 78227-5301, USA. ${ }^{4}$ School of Biological Sciences, Victoria University of Wellington, Wellington 6140, New Zealand. ${ }^{5}$ South Texas Diabetes and Obesity Institute, University of Texas, Rio Grande Valley School of Medicine, Brownsville, TX 78520, USA.

Received: 2 June 2015 Accepted: 2 November 2015

Published online: 02 December 2015

\section{References}

1. Macgregor S, Bellis C, Lea RA, Cox H, Dyer T, Blangero J, et al. Legacy of mutiny on the Bounty: founder effect and admixture on Norfolk Island. Eur J Hum Genet. 2010;18:67-72.

2. McEvoy BP, Zhao ZZ, Macgregor S, Bellis C, Lea RA, Cox H, et al. European and Polynesian admixture in the Norfolk Island population. Heredity (Edinb) 2010;105(2):229-234

3. Benton MC, Stuart S, Bellis C, Macartney-Coxson D, Eccles D, Curran JE, et al. "Mutiny on the Bounty": the genetic history of Norfolk Island reveals extreme gender-biased admixture. Investig Genet. 2015;6:11.

4. Bellis C, Cox HC, Dyer TD, Charlesworth JC, Begley KN, Quinlan S, et al. Linkage mapping of CVD risk traits in the isolated Norfolk Island population. Hum Genet. 2008:124:543-52.

5. Cox HC, Bellis C, Lea RA, Quinlan S, Hughes R, Dyer T, et al. Principal Component and linkage analysis of cardiovascular risk traits in the Norfolk Isolate. Hum Hered. 2009;68:55-64.

6. Maher BH, Lea RA, Benton M, Cox HC, Bellis C, Carless M, et al. An X chromosome association scan of the Norfolk Island genetic isolate provides evidence for a novel migraine susceptibility locus at Xq12. PLoS One. 2012; $7: \mathrm{e} 37903$

7. Benton MC, Lea RA, Macartney-Coxson D, Carless MA, Göring HH, Bellis C, et al. Mapping eQTLs in the Norfolk Island Genetic Isolate Identifies Candidate Genes for CVD Risk Traits. Am J Hum Genet. 2013;93:1087-99.

8. Bellis C, Hughes RM, Begley KN, Quinlan S, Lea RA, Heath SC, et al. Phenotypical characterisation of the isolated Norfolk Island population focusing on epidemiological indicators of cardiovascular disease. Hum Hered. 2006;60:211-9.

9. Chen L, Magliano DJ, Balkau B, Colagiuri S, Zimmet PZ, Tonkin AM, et al. Ausdrisk: an australian type 2 diabetes risk assessment tool based on demographic, lifestyle and simple anthropometric measures. Med J Aust. 2010;192:197-202.

10. Stocker R, Yamamoto Y, McDonagh A, Glazer A, Ames B. Bilirubin is an antioxidant of possible physiological importance. Science (80-). 1987;235:1043-6.

11. Yamaguchi T, Terakado M, Horio F, Aoki K, Tanaka M, Nakajima H. Role of bilirubin as an antioxidant in an ischemia-reperfusion of rat liver and induction of heme oxygenase. Biochem Biophys Res Commun. 1996:223:129-35.

12. Baranano DE, Rao M, Ferris CD, Snyder SH. Biliverdin reductase: a major physiologic cytoprotectant. Proc Natl Acad Sci U S A. 2002;99:16093-8.

13. Cheriyath P, Gorrepati VS, Peters I, Nookala V, Murphy ME, Srouji N, et al. High Total Bilirubin as a Protective Factor for Diabetes Mellitus: An Analysis of NHANES Data From 1999-2006. J Clin Med Res. 2010;2:201-6.

14. Cox AJ, Ng MC-Y, Xu J, Langefeld CD, Koch KL, Dawson PA, et al. Association of SNPs in the UGT1A gene cluster with total bilirubin and mortality in the diabetes heart study. Atherosclerosis. 2013;229:155-60

15. Grant DJ, Bell DA. Bilirubin UDP-glucuronosyltransferase 1A1 gene polymorphisms: susceptibility to oxidative damage and cancer? Mol Carcinog. 2000:29:198-204

16. Melton PE, Haack K, Göring HH, Laston S, Umans JG, Lee ET, et al. Genetic influences on serum bilirubin in American Indians: The Strong Heart Family Study. Am J Hum Biol. 2011;23:118-25. 
17. Chen G, Ramos E, Adeyemo A, Shriner D, Zhou J, Doumatey AP, et al. UGT1A1 is a major locus influencing bilirubin levels in African Americans. Eur J Hum Genet. 2012;20:463-8.

18. Jylhävä J, Lyytikäinen L-P, Kähönen M, Hutri-Kähönen N, Kettunen J, Viikari $J$, et al. A genome-wide association study identifies UGT1A1 as a regulator of serum cell-free DNA in young adults: the cardiovascular risk in young finns study. PLoS One. 2012;7:e35426.

19. Abbasi A, Deetman PE, Corpeleijn E, Gansevoort RT, Gans ROB, Hillege HL, et al. Bilirubin as a potential causal factor in type 2 diabetes risk: a Mendelian randomization study. Diabetes. 2015;64:1459-69.

20. Schwertner $H$, Jackson W, Tolan G. Association of low serum concentration of bilirubin with increased risk of coronary artery disease. Clin Chem. 1994;40:18-23.

21. Madhavan M, Wattigney WA, Srinivasan SR, Berenson GS. Serum bilirubin distribution and its relation to cardiovascular risk in children and young adults. Atherosclerosis. 1997;131:107-13.

22. Novotný $L$, Vítek $L$. Inverse relationship between serum bilirubin and atherosclerosis in men: a meta-analysis of published studies. Exp Biol Med (Maywood). 2003;228:568-71.

23. Schwertner HA. Association of smoking and low serum bilirubin antioxidant concentrations 1 the views expressed in this article are those of the author and do not reflect the official policy of the Department of Defense or other Departments of the US Government 1. Atherosclerosis. 1998;136:383-7.

24. Hoydonck PGV. Serum bilirubin concentration in a Belgian population: the association with smoking status and type of cigarettes. Int J Epidemiol. 2001;30:1465-72

25. Jo J, Kimm H, Yun JE, Lee KJ, Jee SH. Cigarette smoking and serum bilirubin subtypes in healthy Korean men: the Korea Medical Institute study. J Prev Med Public Health. 2012:45:105-12.

26. Vitek $L$. The role of bilirubin in diabetes, metabolic syndrome, and cardiovascular diseases. Front Pharmacol. 2012:3:55

27. McArdle PF, Whitcomb BW, Tanner K, Mitchell BD, Shuldiner AR, Parsa A. Association between bilirubin and cardiovascular disease risk factors: using Mendelian randomization to assess causal inference. BMC Cardiovasc Disord. 2012;12:16.

28. Lin J-P, Schwaiger JP, Cupples LA, O'Donnell CJ, Zheng G, Schoenborn V, et al. Conditional linkage and genome-wide association studies identify UGT1A1 as a major gene for anti-atherogenic serum bilirubin levels-the Framingham Heart Study. Atherosclerosis. 2009;206:228-33.

29. Wu Y, Li M, Xu M, Bi Y, Li X, Chen Y, et al. Low serum total bilirubin concentrations are associated with increased prevalence of metabolic syndrome in Chinese. J Diabetes. 2011;3:217-24.

30. Andersson C, Weeke P, Fosbøl EL, Brendorp B, Køber L, Coutinho W, et al. Acute effect of weight loss on levels of total bilirubin in obese, cardiovascular high-risk patients: an analysis from the lead-in period of the Sibutramine Cardiovascular Outcome trial. Metabolism. 2009:58:1109-15.

31. Guzek M, Jakubowski Z, Bandosz P, Wyrzykowski B, Smoczyński M, Jabloiska A, et al. Inverse association of serum bilirubin with metabolic syndrome and insulin resistance in Polish population. Przegląd Epidemiol. 2012;66:495-501.

32. Choi $\mathrm{SH}$, Yun KE, Choi HJ. Relationships between serum total bilirubin levels and metabolic syndrome in Korean adults. Nutr Metab Cardiovasc Dis. 2013;23:31-7.

33. Kwon K-M, Kam J-H, Kim M-Y, Kim M-Y, Chung CH, Kim J-K, et al. Inverse association between total bilirubin and metabolic syndrome in rural korean women. J Womens Health (Larchmt). 2011;20:963-9.

34. Jo J, Yun JE, Lee H, Kimm H, Jee SH. Total, direct, and indirect serum bilirubin concentrations and metabolic syndrome among the Korean population. Endocrine. 2011;39:182-9.

35. Tephly TR, Burchell B. UDP-glucuronosyltransferases: a family of detoxifying enzymes. Trends Pharmacol Sci. 1990;11:276-9.

36. Burchell B, Brierley $\mathrm{CH}$, Rance D. Specificity of human UDPGlucuronosyltransferases and xenobiotic glucuronidation. Life Sci. 1995:57:1819-31.

37. Fisher MB, Paine MF, Strelevitz TJ, Wrighton SA: The role of hepatic and extrahepatic UDP-glucuronosyltransferases in human drug metabolism. Drug Metab Rev. 2002;33(3-4):273-97

38. Lin J-P, Cupples LA, Wilson PWF, Heard-Costa N, O'Donnell CJ. Evidence for a gene influencing serum bilirubin on chromosome $2 q$ telomere: a genomewide scan in the Framingham study. Am J Hum Genet. 2003;72:1029-34.

39. Newton-Cheh C, Johnson T, Gateva V, Tobin MD, Bochud M, Coin L, et al. Genome-wide association study identifies eight loci associated with blood pressure. Nat Genet. 2009:41:666-76.
40. Johnson AD, Kavousi M, Smith AV, Chen M-H, Dehghan A, Aspelund T, et al. Genome-wide association meta-analysis for total serum bilirubin levels. Hum Mol Genet. 2009;18:2700-10.

41. Bielinski SJ, Chai HS, Pathak J, Talwalkar JA, Limburg PJ, Gullerud RE, et al. Mayo Genome Consortia: a genotype-phenotype resource for genome-wide association studies with an application to the analysis of circulating bilirubin levels. Mayo Clin Proc. 2011;86:606-14.

42. Dai $X$, Wu C, He Y, Gui L, Zhou L, Guo H, et al. A genome-wide association study for serum bilirubin levels and gene-environment interaction in a Chinese population. Genet Epidemiol. 2013;37:293-300.

43. Lingenhel A, Kollerits B, Schwaiger JP, Hunt SC, Gress R, Hopkins PN, et al. Serum bilirubin levels, UGT1A1 polymorphisms and risk for coronary artery disease. Exp Gerontol. 2008;43:1102-7.

44. Bosma PJ, Chowdhury NR, Goldhoorn BG, Hofker MH, Oude Elferink RP, Jansen $\mathrm{PL}$, et al. Sequence of exons and the flanking regions of human bilirubin-UDP-glucuronosyltransferase gene complex and identification of a genetic mutation in a patient with Crigler-Najjar syndrome, type I. Hepatol. 1992;15:941-7

45. Seppen J, Bosma PJ, Goldhoorn BG, Bakker CT, Chowdhury JR, Chowdhury NR, et al. Discrimination between Crigler-Najjar type I and II by expression of mutant bilirubin uridine diphosphate-glucuronosyltransferase. J Clin Invest. 1994;94:2385-91.

46. Kadakol A, Ghosh SS, Sappal BS, Sharma G, Chowdhury JR, Chowdhury NR Genetic lesions of bilirubin uridine-diphosphoglucuronate glucuronosyltransferase (UGT1A1) causing Crigler-Najjar and Gilbert syndromes: correlation of genotype to phenotype. Hum Mutat. 2000;16:297-306.

47. Black M, Billing BH. Hepatic bilirubin udp-glucuronyl transferase activity in liver disease and gilbert's syndrome. N Engl J Med. 1969;280:1266-71.

48. Koiwai O, Nishizawa M, Hasada K, Aono S, Adachi Y, Mamiya N, et al. Gilbert's syndrome is caused by a heterozygous missense mutation in the gene for bilirubin UDP-glucuronosyltransferase. Hum Mol Genet. 1995;4:1183-6.

49. Borlak J, Thum T, Landt O, Erb K, Hermann R. Molecular diagnosis of a familial nonhemolytic hyperbilirubinemia (Gilbert's syndrome) in healthy subjects. Hepatol. 2000;32(4 Pt 1):792-5.

50. Bosma PJ, Chowdhury JR, Bakker C, Gantla S, de Boer A, Oostra BA, et al. The genetic basis of the reduced expression of bilirubin UDP-glucuronosyltransferase 1 in Gilbert's syndrome. N Engl J Med. 1995;333:1171-5.

51. Bulmer AC, Blanchfield JT, Toth I, Fassett RG, Coombes JS. Improved resistance to serum oxidation in Gilbert's syndrome: A mechanism for cardiovascular protection. Atherosclerosis. 2008;199:390-6.

52. Inoguchi T, Sasaki S, Kobayashi K, Takayanagi R, Yamada T. Relationship between Gilbert syndrome and prevalence of vascular complications in patients with diabetes. JAMA. 2007:298:1398-400.

53. Dekker D, Dorresteijn MJ, Pijnenburg M, Heemskerk S, Rasing-Hoogveld A, Burger DM, et al. The bilirubin-increasing drug atazanavir improves endothelial function in patients with type 2 diabetes mellitus. Arterioscler Thromb Vasc Biol. 2011:31:458-63.

54. Beutler E, Gelbart T, Demina A. Racial variability in the UDPglucuronosyltransferase 1 (UGT1A1) promoter: a balanced polymorphism for regulation of bilirubin metabolism? Proc Natl Acad Sci. 1998;95:8170-4

55. Frazer KA, Ballinger DG, Cox DR, Hinds DA, Stuve LL, Gibbs RA, et al. A second generation human haplotype map of over 3.1 million SNPs. Nature. 2007:449:851-61.

56. Oussalah A, Bosco P, Anello G, Spada R, Guéant-Rodriguez R-M, Chery C, et al. Exome-wide association study identifies new low-frequency and rare UGT1A1 coding variants and UGT1A6 coding variants influencing serum bilirubin in elderly subjects: a strobe compliant article. Medicine (Baltimore) 2015:94:e925.

57. Bellis C, Cox HC, Ovcaric M, Begley KN, Lea RA, Quinlan S, et al. Linkage disequilibrium analysis in the genetically isolated Norfolk Island population. Heredity (Edinb). 2008;100:366-73.

58. Aulchenko YS, Ripke S, Isaacs A, Van Duijn CM. GenABEL: an R library for genorne-wide association analysis. Bioinformatics. 2007;23:1294-6.

59. Scharpf RB, Irizarry RA, Ritchie ME, Carvalho B, Ruczinski I. Using the R package crlmm for genotyping and copy number estimation. J Stat Softw. 2011;40:1-32.

60. Purcell S, Neale B, Todd-Brown K, Thomas L, Ferreira MAR, Bender D, et al. PLINK: a tool set for whole-genome association and population-based linkage analyses. Am J Hum Genet. 2007;81:559-75.

61. Manichaikul A, Mychaleckyj JC, Rich SS, Daly K, Sale M, Chen WM. Robust relationship inference in genome-wide association studies. Bioinformatics. 2010;26:2867-73. 
62. O'Connell J, Gurdasani D, Delaneau O, Pirastu N, Ulivi S, Cocca M, et al. A general approach for haplotype phasing across the full spectrum of relatedness. PLoS Genet. 2014;10:e1004234.

63. Barrett JC, Fry B, Maller J, Daly MJ. Haploview: analysis and visualization of LD and haplotype maps. Bioinformatics. 2005;21:263-5.

64. R-Development-Core-Team: R: A language and environment for statistical computing. 2015.

Submit your next manuscript to BioMed Central and take full advantage of:

- Convenient online submission

- Thorough peer review

- No space constraints or color figure charges

- Immediate publication on acceptance

- Inclusion in PubMed, CAS, Scopus and Google Scholar

- Research which is freely available for redistribution 